\title{
Numerical investigation of vibrational properties and study of some selected physical properties of potassium chloride $(\mathrm{KCl})$
}

\begin{abstract}
In this work, we investigate the vibrational properties of diatomic $\mathrm{KCl}$ lattice, the dispersion relation, density of states and the specific heat capacity were obtained using a quantum approach analytically, a simulated FOTRAN-90 code program was used to evaluate these properties numerically. The frequencies of the three normal modes at equal intervals of $\mathrm{k}=$ $\pm \mathrm{n} \pi / \mathrm{a}$ (wave vector), along the three symmetry directions [001], [011] and [111] of $\mathrm{KCl}$ lattice were calculated. The subroutine was designed to give the output in numerical values that can be drawn graphically using any form of graphical tools, and the result obtained shows an excellent agreement with the many results obtained from other means.
\end{abstract}

Keyword: Dispersion relation of crystal; Potassium chloride; Quantum approach of dynamical matrix; Specific heat capacity 